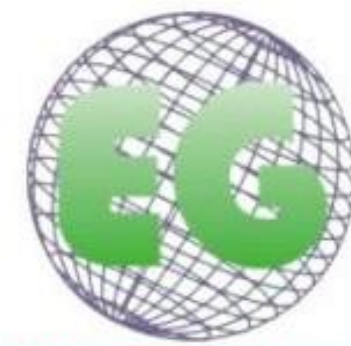

ISSN 1695-6141 $N^{\circ} 51$

\title{
Violencia de Género: Papel de Enfermería en la Prevención Secundaria desde Atención Primaria
}

Role of Nursing in Secondary Prevention from Primary Care

\author{
Raquel Baides Noriega ${ }^{1}$ \\ ${ }^{1}$ Enfermera del Servicio de Salud del Principado de Asturias (SESPA). Asturias.España. \\ E-mail: Rakel noriega1991@outlook.es
}

http://dx.doi.org/10.6018/eglobal.17.3.307241

Recibido: $18 / 10 / 2017$

Accepted: 07/12/2017

\section{RESUMEN:}

Introducción: La violencia de género es un fenómeno complejo que se relaciona con la desigualdad de género presente en todas las culturas y tiene múltiples consecuencias para la salud. Se trata de un problema de salud pública en el que la Enfermería puede realizar un importante papel de prevención secundaria.

Metodología: Con objeto de conocer procedimientos para la detección e intervención precoz de la violencia de género desde Enfermería de Atención Primaria se realizó una revisión bibliográfica a través de fuentes primarias y secundarias.

Resultados: La prevención secundaria de la violencia de género es una tarea imprescindible desde los equipos de atención primaria. Los indicadores de VG presentes en la literatura son diversos, y de su detección depende la posibilidad de intervenir. La identificación de casos se basa en la relación de confianza y en técnicas como la entrevista o el uso de cuestionarios. Los procedimientos establecidos ante su confirmación están regulados por diferentes normas legales y deontológicas.

Conclusión: La violencia de género es un grave problema que afecta, de forma importante, a la salud de las mujeres que la sufren. Enfermería de Atención Primaria juega un papel fundamental por su gran accesibilidad y contacto frecuente con la mujer a lo largo del ciclo vital, pudiendo detectar precozmente violencia de género. Para potenciar la prevención secundaria, se hace preciso mejorar la formación entre los profesionales sanitarios.

Palabras clave: Violencia; Género; Enfermería; Detección; Prevención; Secundaria.

\section{ABSTRACT:}

Introduction: Violence against women is a complex phenomenon that is related to the present in all cultures gender inequality and has on instrumental character. It's a public health problem in which nurses can perform a important role of secondary prevention.

Methodology: In order to learn about the phenomenon of the gender violence and the procedures for detection and early intervention from primary care nursing, we conducer a literature review through primary and secondary sources.

Results: Secondary prevention of gender-based violence is an essential task from the primary care teams. Indicators of suspicion in the literature are diverse, and its detection depends on the possibility 
of intervention. The identification of cases is based on the relationship of trust and in techniques such as the use of questionnaires of interview. Before his confirmation procedures are regulated by different legal and deontological rules.

Conclusions: Gender-based violence is a serious problem which affects, significantly, to the health of women suffers it. Primary care nursing plays an essential role, since it possesses great accessibility and direct contact with the women throughout the life cycle, being able to detect early gender-based violence. To promote secondary prevention, is necessary to improve the training among heath professionals.

Keywords: Violence; Gender; Nursing; Detection; Secondary prevention.

\section{INTRODUCCIÓN}

La violencia de género o violencia contra la mujer (VG en adelante), es un fenómeno complejo, confuso y heterogéneo. Se ha definido (ONU, 1996) como: "Todo acto que resulte, o pueda tener como resultado un daño físico, psicológico o sexual para la mujer, inclusive las amenazas de tales actos, la coacción o la privación arbitraria de libertad, tanto si se producen en la vida pública como en la privada"(1).

La magnitud del fenómeno a nivel mundial es muy importante, basta considerar los datos de la OMS de $2016^{(2)}$, según los cuales la prevalencia de VG física, psicológica y sexual, fue del $35 \%$. Además la VG fue la primera causa de muerte de mujeres entre los 15 y los 44 años de edad superando la suma de las provocadas por cáncer, malaria, accidentes de tráfico y guerras, según la OMS ${ }^{(1)}$.

La VG se puede producir en cualquier ámbito, sin embargo en pareja y familia son los contextos más frecuentes ${ }^{(3)}$. Existen diferentes tipos de VG (física, psicológica, sexual, económica...) y niveles de gravedad (desde un insulto machista al asesinato ${ }^{(4)}$. Se ha descrito como un fenómeno "iceberg" en el cual la mayor parte del mismo se encuentra invisibilizado socialmente, dándose visibilidad únicamente a los casos graves de VG.

La VG produce graves consecuencias para la salud de la mujer y del núcleo familiar, siendo especialmente relevantes las consecuencias para la salud de los hijos que viven alrededor del maltrato ${ }^{(4)}$. Los problemas de salud que produce la VG determinan que la mujer acuda con más frecuencia a los servicios de Atención Primaria ${ }^{(1)}$.

La Atención Primaria (AP), forma el primer nivel asistencial, y se caracteriza por tener alta accesibilidad, capacidad para abordar íntegramente problemas de salud y atender a la comunidad de una forma integral, integrada, permanente, continuada y activa ${ }^{(5)}$. La Enfermería de AP, abarca la atención de todas las personas, enfermas o no, incluyendo actividades de prevención de la enfermedad, mantenimiento y recuperación de la salud ${ }^{(5)}$. La inversión en prevención de VG es altamente eficiente para el servicio de salud ${ }^{(4)}$.

En el campo de la prevención encontramos la prevención secundaria, que consiste en detectar precozmente el problema y aplicar intervenciones dirigidas a impedir 0 retrasar el desarrollo de enfermedades. La prevención secundaria es una tarea fundamental dentro de los equipos de atención primaria ${ }^{(5)}$. Los Centros de Salud (CS en adelante) son lugares privilegiados para ello, considerando que frecuentemente las mujeres que sufren VG acuden a los $C S^{(12)}$. Según Cirici et al ${ }^{(3)}$, aproximadamente el 
90\% de mujeres maltratadas acude al centro de AP durante el año siguiente de haber sido agredida.

Sin embargo, se ha mostrado infradetección desde los servicios sanitarios de la VG, solo se diagnostica un pequeño porcentaje con un retraso de entre 6 y 10 años desde el inicio de las agresiones ${ }^{(4)}$.

El abordaje este tema desde el servicio sanitario de AP es imprescindible para dar una adecuada respuesta al problema de la VG y llevar acabo un modelo interdisciplinario de atención a la comunidad ${ }^{(5)}$.

\section{OBJETIVOS}

Con el presente trabajo se pretende fortalecer el conocimiento sobre las pautas de actuación para la prevención secundaria de la VG desde Enfermería de AP, para estar en condiciones de abordar de una forma más integral la situación y adquirir un papel activo.

\section{METODOLOGÍA}

Para alcanzar los objetivos propuestos se ha realizado una revisión bibliográfica rigurosa y reproducible sobre el fenómeno de la VG. Se abordan aspectos relevantes para detectar la VG incidiendo especialmente en la detección y abordaje precoz o prevención secundaria desde Atención primaria.

Para la búsqueda de documentos se han utilizado los siguientes recursos:

Bases de datos científicas: Cuiden, PubMed, Scielo y Science Direct.

$>$ Repositorios de Salud: Elsevier.

Para la búsqueda de información, a través de estos recursos, se ha utilizado lenguaje controlado. El lenguaje controlado (DeCS-MeSH), se ha empleado para la revisión de las bases de datos científicas de salud, utilizando las palabras clave empleadas en el presente trabajo, y los boléanos "and" y "or" como mediadores entre ellos. Las palabras clave han sido: Enfermería, violencia, género, detección, prevención secundaria.

Los criterios de inclusión en la búsqueda han sido selección de artículos de los últimos 10 años (2008-2017), priorizando aquellos con menos de 5 años de antigüedad, revisando artículos en idioma español, inglés y portugués y descartando aquellos que no poseen relevancia respecto el tema a tratar. Se ha dado prioridad a la temática enfermera, y especialmente, a documentos relacionados con el ámbito en la AP.

Tras la búsqueda de información con los criterios de inclusión establecidos y el uso para la búsqueda de información de las palabras llave, se han obtenido 574 artículos que se han revisado a nivel de título y resumen, descartando aquellos sin suficiente relevancia o calidad. Para la elaboración del presente trabajo se han utilizado 13 artículos.

Además, para la elaboración del trabajo, se han utilizado fuentes primarias, como el Código Deontológico de Enfermería: Resolución 32/89 y el libro de Enfermería Comunitaria $^{(5)}$. 
También se han obtenido documentos de interés mediante lenguaje libre, en las Páginas Webs de Organismos Oficiales Internacionales, Nacionales y Locales: Organización Mundial de la Salud (OMS-WHO), Observatorio Estatal de Violencia sobre la Mujer (Ministerio de Sanidad, Servicios Sociales e Igualdad), Fisterra, Portal de Salud del Principado de Asturias (AsturSalud). Se destaca el Protocolo Nacional Común Sanitario para la atención a la Violencia de Género, el Protocolo Interdepartamental de Asturias para la atención a Violencia de género: ámbito sanitario.

\section{RESULTADOS}

\section{Prevención secundaria de la VG desde Enfermería de AP}

La prevención secundaria, detección y abordaje precoz es una tarea fundamental dentro de los equipos de atención primaria ${ }^{(5)}$. La inversión en prevención de VG es altamente eficiente para el servicio de salud, como ha mostrado un estudio australiano ${ }^{(6)}$, cuyo principal hallazgo fue que la disminución de 5 puntos porcentuales de prevalencia en VG podría producir un ahorro de 377 millones de dólares, pudiendo llegar a los 2.000 millones de dólares si la VG se redujera a cero.

Los Centros de Salud (CS en adelante) de AP son lugares privilegiados para ello, considerando que aproximadamente el $30 \%$ de las mujeres que acuden a los CS están sufriendo violencia por parte de su pareja ${ }^{(3)}$. Sin embargo, desde los servicios sanitarios solo se diagnostica un pequeño porcentaje con un retraso de entre 6 y 10 años desde el inicio de las agresiones ${ }^{(4)}$.

La infradetección desde los servicios sanitarios se ha relacionado con diferentes factores que la obstaculizan ${ }^{(4,7,8)}$. Sin embargo, entre todas las dificultades para detectar la VG, los profesionales sanitarios destacan la falta de formación y de conocimientos como principal motivo de infadetección/actuación ${ }^{(9,10,11)}$.

La formación se considera fundamental, ya que tener 21 horas o más de formación se ha asociado con mayor probabilidad en los profesionales de indagar sobre VG, la probabilidad aumenta progresivamente con la formación avanzada ${ }^{(12)}$. Sin embargo la falta de formación de los profesionales sanitarios para abordar el problema se demuestra en numerosos estudios ${ }^{(12-14)}$.

\section{Indicadores de sospecha de VG}

Para poder detectar la VG debemos conocer los Indicadores de Sospecha de la misma ${ }^{(4)}$ :

> Mujer con antecedentes como: maltrato infantil o violencia en la familia.

> Hábitos de vida como el uso de alcohol, drogas o psicofármacos.

> La presentación de las repercusiones en la salud de la VG pueden actuar como indicadores de sospecha de VG

> En caso de embarazo, puerperio y/o problemas ginecobstétricos: lesiones abdominales sin justificación adecuada, inicio tardío en visitas de control prenatal, depresión post parto que no remite, demanda frecuente de anticonceptivos de emergencia ${ }^{(4,8)}$

> Ante lesiones físicas evidentes: relato incoherente del mecanismo lesivo, alta frecuencia de incidentes, lesiones de diferente estadio evolutivo o crisis de ansiedad $(4,19)$. 
$>$ Mujer que se comporta de la siguiente forma: mirada huidiza, ropa inadecuada a la época del año, nerviosa, falta de cuidado personal, actitudes de aceptación de la violencia. El comportamiento de la mujer cuando acude con su pareja a la consulta suele ser con temor al responder, mira a su pareja antes de hablar y busca constantemente su aprobación ${ }^{(4,8)}$.

$>$ La utilización de los servicios sanitarios por estas mujeres se caracteriza por alternar periodos de híper frecuentación con otros de abandono, incumplimiento de citas, uso repetitivo de los servicios de urgencias, frecuentes hospitalizaciones, acudir en pareja cuando antes no lo hacía ${ }^{(8)}$.

$>$ Hombre que acompaña a su pareja y se comporta de la siguiente forma: es la pareja la que explica los síntomas de la enfermedad de la mujer, controla la medicación, solicita estar presente en todas las visitas de atención primaria, además desvaloriza las capacidades de la mujer y suele ser agresivo con el equipo sanitario solicitando atención para ella ${ }^{(4)}$.

La acumulación de indicadores de sospecha de VG nos debe hacer sospechar de situación de maltrato ${ }^{(4)}$.

\section{Detección Precoz}

La detección precoz de la VG en las primeras fases es muy importante, ya que tanto las consecuencias físicas como psicológicas serán menores (4). El Programa de Actividades Preventivas de Salud (PAPPS) no recomienda el cribado poblacional pero sí tomar postura activa y mantener pautas de actuación (1). Sin embargo, los protocolos españoles señalan que en toda mujer mayor de 14 años debe valorarse la posibilidad de VG y recoger en su historia clínica la posibilidad maltrato ${ }^{(4,8)}$.

Para detectar la VG de un modo oportunista se pueden plantear preguntas de abordaje psicosocial del siguiente tipo, "dada la alta frecuencia 0 las graves consecuencias para la salud de maltrato, actualmente preguntamos a todas las mujeres la posibilidad del mismo" (4).

Entre las escalas de cribado de VG aplicables a nuestra población, destaca la siguiente por su sencillez y rapidez para detectar riesgo del problema en el escaso tiempo de las consultas ${ }^{(1)}$ : - Woman Abuse Screening Tool: consta de dos preguntas adaptadas a la población española.

Tabla 1: Woman Abuse Screening Tool. Elaboración Propia. Fuente: Adaptación de la Guía de Práctica Clínica sobre VG de Menéndez et al ${ }^{(1)}$.

\begin{tabular}{|l|l|l|l|}
\hline 1- En general, ¿Cómo describiría usted su relación de pareja? \\
\hline Mucha tensión (1punto) & Alguna tensión (0 pto.) & Sin tensión (0 pto.) \\
\hline 2- Usted y su pareja resuelven sus discusiones con: \\
\hline $\begin{array}{l}\text { Mucha dificultad } \\
\text { (1punto) }\end{array}$ & $\begin{array}{l}\text { Alguna dificultad } \\
\text { pto.) }\end{array}$ & Sin dificultad (0 pto.) \\
\hline
\end{tabular}

Existen dos maneras de puntuar esta escala: la más aceptada según Juncal, Hernández y Ruiz ${ }^{(15)}$, es dar una puntuación de 1 a las respuestas más externas de "mucha tensión" y "mucha dificultad" y 0 a las demás opciones de respuesta. Una puntuación de 1 se considera positiva en el cribado, o lo que es lo mismo, alto riesgo 
de VG. La fiabilidad de este cuestionario se ha considerado alta tras la validación del mismo ${ }^{(16)}$. El cuestionario de esta escala se presenta en el Anexo I.

Existen diferentes instrumentos para la detección de la VG, como la entrevista personal a la mujer y escalas de detección de VG ${ }^{(4)}$. Cada profesional debe buscar su forma personal de abordar la situación, no existiendo un patrón ideal ${ }^{(1)}$. La OMS, en su informe VCM recomienda al personal sanitario que: "No tenga miedo de preguntar: contrariamente a la creencia popular, la mayoría de las mujeres están dispuestas a revelar el maltrato cuando se les pregunta en forma directa y no valorativa. En realidad, muchas están esperando silenciosamente que alguien les pregunte" (4).

La entrevista es el proceso comunicativo entre el paciente y el profesional. Es fundamental crear un clima de comunicación abierto, actitud empática y escucha activa que facilite la comunicación. Se deben evitar las actitudes paternalistas, de culpabilidad hacia la mujer, o darla a entender que la situación se va a resolver fácilmente. Además es muy importante cuidar la comunicación no verbal, observar las actitudes y emociones facilitando la expresión de sentimientos ${ }^{(4,8)}$.

Debe seguirse una secuencia lógica de preguntas más generales e indirectas a otras más concretas y directas, pero abordando directamente el tema de la violencia y defendiendo la no justificación de este tipo de conductas en las relaciones humanas. Como norma general para la protección de la victima nunca debemos verificar el testimonio hablando con su agresor, además en caso de que la mujer acuda a la consulta con hijos/as, estos deberán permanecer en la sala de espera ${ }^{(4,8)}$.

Cuando la entrevista se dirige a valorar la presencia de maltrato ante indicadores de sospecha presentes en la mujer, existen una serie de preguntas tipo que pueden ser de ayuda para los profesionales de enfermería. A continuación, se muestran ejemplos de preguntas tipo que nos ofrece el protocolo nacional común sanitario para la atención a la VG ${ }^{(8)}$ :

> "He repasado su historial y encuentro algunas cosas que me gustaría comentar con usted. Veo que: (relatar los hallazgos), ¿a qué cree que se debe su malestar ó problema de salud?, La encuentro algo intranquila ¿qué le preocupa? ¿Está viviendo alguna situación problemática que le haga sentirse así?, ¿qué me puede decir a esto? ¿Cree que todo está relacionado?"

$>$ Ante sospecha por lesión física: "esta lesión sucede cuando se recibe un golpe, empujón, puñetazo, corte, ¿eso es lo que ha ocurrido?”, ¿su pareja ha utilizado la fuerza contra usted?, ¿cómo?, ¿desde cuándo? ",” ¿alguna vez la ha agredido más gravemente?"

> En el caso de sospecha por los síntomas o problemas psíquicos encontrados: "Me gustaría conocer su opinión sobre esos síntomas que me ha contado: ¿Desde cuándo se siente usted así? ¿A qué cree usted que se deben? ¿Los relaciona con algo? ¿Tiene alguna dificultad para ver a sus amistades o familiares? ¿Qué le impide hacerlo?"

Cuando el personal sanitario sospeche de una situación de VG, debe confirmar o descartar dicha conjetura. Es importante dejar claro que toda relación saludable está basada en la confianza y el respeto mutuo ${ }^{(4)}$. Los primeros signos de maltrato pueden ayudar a las mujeres a reconocer la situación, los cuales suelen expresarse mediante los siguientes comportamientos de la pareja hacia la mujer: ridiculizar, 
aislar, humillar, gritar, insultar, culpabilizar, controlar (dinero, forma de vestir, teléfono móvil, redes sociales), amenazar en caso de separación.

Las nuevas tecnologías, especialmente entre personas jóvenes, pueden ser un medio para ejercer o recibir VG. Según Ivethe et al ${ }^{(17)}$, entre el 68 y el $92 \%$ de los adolescentes españoles manifestaron conductas de celos, control e intrusión en este contexto. Por lo que se deben valorar las redes sociales y nuevas tecnologías como medio de ejercer y recibir violencia, especialmente psicológica en personas jóvenes. Además existen escalas para detectar la VG y su nivel de intensidad, como por ejemplo ${ }^{(1)}$ :

- La versión española de Index Of. Spouse Abuse (ISA): formada por 30 ítems, mide violencia física, sexual y emocional, conductas de control y aislamiento y la gravedad de la violencia contra la mujer, útil para realizar estudios más profundos de investigación sobre la violencia.

- El PMWI-SF, mide el grado de maltrato psicológico ${ }^{(1)}$. Este cuestionario es una versión corta del original, incluye comportamientos abusivos que pueden sufrir las mujeres víctimas de VG.

Tanto si la mujer reconoce sufrir malos tratos, como si presenta indicadores de sospecha pero no lo reconoce, se deben realizar una serie de valoraciones ${ }^{(1,8)}$. Como norma general, los profesionales, nunca se debe criticar ausencia de respuesta de la víctima, con frases como "Si usted quisiera se iría..." ni recomendar terapia de pareja ${ }^{(4)}$.

Ante detección del mismo por parte del personal sanitario pero negación de violencia por parte de la mujer, debemos intentar suprimir el miedo a la revelación del abuso y hacer entender a la mujer que no es culpable de la violencia que sufre, ayudarla a tomar decisiones satisfactorias pero sin imponer, alertando de los riesgos de aceptar su situación ${ }^{(4)}$. Algunas intervenciones que ayudan a reconocerlo son: informar sobre los recursos existentes, recordar que el maltrato es un problema de salud y que puede contar con el equipo sanitario en ese momento o para futuras consultas.

La valoración integral de la situación incluirá 3 valoraciones ${ }^{(1,4)}$ :

1. Valoración Biopsicosocial: debe incluir una exploración de lesiones, síntomas físicos y situación, familiar, emocional y/o económica.

2. Valoración de la situación de violencia: Tipo, frecuencia, intensidad de VG, tiempo de evolución y alcance de salud, afectación a otros miembros del núcleo familiar, mecanismos de afrontamiento desarrollados por la mujer y fase del proceso de motivación para el cambio en el que se encuentra la víctima.

3. Valoración de seguridad y riesgo vital: se realiza para valorar riesgo de lesiones graves o peligro de vida para la mujer, sus hijos/as o familiares ${ }^{(4)}$.

Para valorar el riesgo es fundamental la sensación de seguridad de la mujer, por lo que consideramos la situación como de riesgo en caso de que la mujer: tenga miedo a regresar a su casa, haya sido amenazada, tanto ella como personas a su cargo, presenta lesiones físicas que manifiestan violencia grave o no cuente con apoyos familiares o sociales. La percepción de peligro por parte de la mujer, tanto para ella como para otros miembros de la familiar, define directamente la situación como de peligro extremo ${ }^{(8)}$. 


\section{Abordaje temprano de la VG}

Para el abordaje del problema se deben seguir los principios éticos profesionales recogidos en la Ley de Autonomía del paciente y el código deontológico de Enfermería ${ }^{(18)}$. Ante todo se deben evitar prejuicios con las intervenciones (principio de no maleficencia), además de velar por el beneficio de la salud (principio de beneficencia) ${ }^{(4)}$. Es importante recordar que el Código Deontológico de la Enfermería Española ${ }^{(18)}$ señala que la Enfermería tiene la obligación de defender los derechos del paciente ante malos tratos físicos o mentales (Art. 55) teniendo un papel primordial en la salvaguarda de los derechos humanos. (Art. 53). También es importante tener presente los derechos y obligaciones en cuanto a la información y documentación clínica (Ley de Autonomía del Paciente y Ley de Protección de Datos). La confidencialidad, privacidad e intimidad, deben mantenerse en toda intervención.

El registro de la información en la historia clínica, como documento con validez legal, puede determinar que la mujer sufre violencia de forma mantenida, por lo que es de especial importancia en estos casos ${ }^{(4)}$.

Se debe respetar la autonomía de la mujer para la toma de decisiones, sin embargo, en España, el personal sanitario tiene la obligación legal de poner en conocimiento la situación de posible hecho delictivo a la autoridad judicial. Los profesionales pueden encontrarse ante el dilema de comunicar la situación o el derecho de autonomía de la mujer, cuando ésta no desea revelar la situación ${ }^{(4)}$. Ante este dilema ético la ley de protección a la mujer de $2004{ }^{(8)}$ establece y especifica que en caso de sospecha de delito, la salvaguarda de la confidencialidad no es una obligación absoluta, teniendo los profesionales sanitarios la obligación de comunicar situaciones de posibles hechos delictivos a las autoridades judiciales.

La finalidad de la intervención es restablecer la salud, y promocionar el desarrollo de una vida en un entorno saludable y libre de violencia.

Tras la confirmación de una sospecha, el personal sanitario debe realizar una función de soporte en educación e información a la mujer, atención en consulta y derivación cuando se requiera. La respuesta de las mujeres ante una situación de violencia de género está condicionada entre otras causas por los recursos psicosociales y apoyos de los que dispone ${ }^{(4)}$. Es probable que cuando las mujeres llegan al CS ya hayan realizado un proceso de reflexión, incluso tomando decisiones, por lo que es importante tener en cuenta el proceso de cambio en el que se encuentra la mujer para no cometer errores en la intervención.

La intervención sanitaria dependerá de la valoración de riesgo vital, cuando ésta resulte negativa, dependerá de la fase de motivación para el cambio en la que se encuentre la mujer ${ }^{(4)}$.

\section{Valoración de riesgo vital negativa}

Cuando la mujer no se encuentra en riesgo, las pautas de atención de referencia ${ }^{(4,8)}$ para el seguimiento en consulta de AP dependerán de la fase para el cambio en la que se encuentre la victima. Las principales pautas de intervención se presentan a continuación ${ }^{(1,4)}$ : 
$>$ Precontemplativa, la mujer no tiene conciencia de su situación: entrevista breve como instrumento para el abordaje, donde dejemos constar nuestra disposición a ayudarla. Se debe ofrecer información para que analice lo que es el maltrato y el buen trato, relacionando las consecuencias para la salud con la situación de violencia ${ }^{(1,4)}$.

> Contemplativa, la mujer comienza a tomar conciencia de su situación: entrevista motivacional como instrumento para el abordaje, la cual se basa en la escucha reflexiva. Se debe analizar el ciclo de la violencia, el impacto en la salud de la VG, poner nombre a su situación para trabajar la toma de conciencia. Mantener consultas de seguimiento para favorecer la toma de decisiones e inicio de cambios ${ }^{(1,4)}$.

> Preparatoria, la mujer se plantea romper con la relación no saludable: entrevista motivacional como instrumento para el abordaje pero con intervenciones diferentes, apoyando iniciativas de cambio, beneficios del mismo y acordando el plan más adecuado a la vez que se analizan dificultades (económicas, familiares, de apoyos, etc. $)^{(1,4)}$.

$>$ De acción, la mujer toma la decisión de ruptura del vínculo: En esta etapa es importante analizar con ella el ciclo de la violencia, incidiendo en la fase de reconciliación como momento clave para recaer en la VG. Se da un importancia extrema a la seguridad en esta fase del ciclo, por ello se debe plantear la elaboración de una estrategia de prevención ante posible situación extrema ${ }^{(4)}$.

$>$ De mantenimiento, consolidado el cambio se plantean nuevos proyectos de vida: Se debe motivar a la mujer a mantener los cambios establecidos, a participar en grupos de apoyo, reevaluando sus avances. En esta fase las recaídas son frecuentes, por lo que es importante potenciar la participación en actividades y redes sociales de la mujer, desarrollando su autoestima y confianza ${ }^{(1,4)}$.

> Recaída: Ante la recaída se debe hacer entender a la mujer que los retrocesos y las inseguridades forman parte del proceso y analizar motivos que llevaron al mismo. Se deben mantener las intervenciones sobre el desarrollo de la autoestima y la confianza, potenciando la participación en actividades sociales ${ }^{(1,4)}$.

El proceso enfermero es individualizado, cada caso será diferente, no existiendo un plan de cuidados estandarizado ${ }^{(19)}$. Para el abordaje integral de victimas de VG se deben atender los problemas físicos, psíquicos y sociales derivados de la situación, de forma integral e interdisciplinar, los cuales pueden ser detectados mediante la Valoración de Enfermería. Debido a las múltiples consecuencias para la salud que tiene el maltrato, existen muchos diagnósticos de Enfermería, según la taxonomía NANDA, aplicables a la VG ${ }^{(19)}$.

Sin embargo, según Holgado, Molina y Pérez ${ }^{(20)}$, los diagnósticos de Enfermería prevalentes entre mujeres víctimas de VG de su estudio, según la taxonomía NANDA, fueron los siguientes: temor, manejo inefectivo del régimen terapéutico, riesgo de suicidio, conflicto de decisiones, baja autoestima situacional y ansiedad.

\section{Valoración de riesgo vital positiva}

Si la valoración de riesgo vital resulta positiva se debe informar a la mujer sobre la situación de peligro en la que se encuentra, sobre el escenario de protección y teléfonos de emergencias en caso de incidencia grave, el 112 (emergencias) y el 016 
(cuerpos policiales). Es de vital importancia conocer la situación de la mujer en cuanto a personas dependientes y apoyos familiares o sociales ${ }^{(8)}$.

Debe primar la seguridad por encima de otras intervenciones por lo que se deberá derivar con carácter urgente al facultativo para que éste emita el correspondiente informe médico al juez y el parte de lesiones si precisa ${ }^{(4)}$.

\section{DISCUSIÓN}

La VG es un importante y complejo problema de salud pública. La detección precoz es fundamental para prevenir posibles consecuencias para la salud del maltrato. Para aumentar la detección precoz de la VG es fundamental la formación de los profesionales sobre el fenómeno y pautas de actuación. Se recomienda que las instituciones faciliten la formación y los profesionales tomen una postura activa para la detección del maltrato. El desarrollo personal de las habilidades sociales y comunicativas se considera fundamental para el manejo adecuado de la entrevista personal tanto para la detección precoz como para el abordaje de la situación.

En este contexto, las instituciones deberán facilitar protocolos y formación completa y consensuada con el resto de organismos y servicios oficiales, ya que, tras la revisión de diferentes protocolos sanitarios españoles, se considera insuficiente la información encontrada respecto a algunos aspectos. Los profesionales sanitarios no estamos capacitados para determinar qué tipos de violencia son delito, por ello no se esclarece el dilema ético sobre el derecho de autonomía de la mujer cuando no desea revelar la situación y la obligación de revelar la situación a las autoridades judiciales ante posible hecho delictivo. Se consideran necesarias normas de actuación al respecto. No se debe olvidar que la confianza depositada por la mujer y la buena calidad del vínculo entre la mujer y la enfermera puede determinar el impacto en la salud de la mujer ${ }^{(4)}$ y verse afectado por este tipo de actuaciones. Por otra parte, la PAPPS no recomienda el cribado poblacional pero sí mantener una postura activa, sin embargo, los protocolos revisados indican que toda mujer mayor de 14 años debe tener registrado en su historia clínica la posibilidad de maltrato, lo cual indica un cribado a dicha edad. Tampoco se establecen unas pautas en cuanto a la periodicidad de las consultas de seguimiento de la mujer maltratada.

Según la información revisada, tras este primer registro sobre la posibilidad de maltrato de la mujer, deberemos mantener una postura activa ante indicadores de sospecha. Se considera que esta pauta es adecuada si los profesionales sanitarios conocen los indicadores de sospecha de VG. Sin embargo, la mayoría de los profesionales no están formados sobre el tema, por lo que se sugiere disponer de unas pautas de periodicidad para el cribado, para ayudar a detectar el maltrato, teniendo en cuenta que según la OMS: "la mayoría de las mujeres están dispuestas a revelar el maltrato cuando se les pregunta en forma directa y no valorativa" (2).

Los profesionales sanitarios, especialmente equipos de atención comunitaria pueden ser claves en la detección y abordaje precoz de los casos ya que, en ocasiones, cuentan con una relación de confianza con las mujeres que no cuentan otros profesionales de otras ramas o disciplinas. Además, la atención a la VG desde Enfermería puede considerarse primordial desde nuestra profesión, ya que, según el código deontológico (18) "La Enfermera/o tendrá como responsabilidad primordial 
profesional la salvaguarda de los Derechos Humanos" (Art. 53), siendo considerada la VG una vulneración de dichos derechos por la ONU ${ }^{(1)}$.

\section{Limitaciones del Estudio}

La complejidad del fenómeno y la escasez de estudios, investigaciones y revisiones sistemáticas sobre la prevención secundaria de la VG, limitan este estudio. Resulta necesaria más investigación en este campo.

\section{CONCLUSIONES}

La violencia de género es un problema de salud pública que consiste en la producción de sufrimiento a la mujer por el hecho de ser mujer, es decir; está causado por la desigualdad de género.

La Enfermería de Atención Primaria, por su gran accesibilidad, cercanía y contacto frecuente con las mujeres a lo largo del ciclo vital supone un colectivo fundamental para llevar a cabo la detección temprana de la violencia de género.

La atención integral a la mujer víctima de VG y las actuaciones de prevención desde Atención Primaria deben contextualizarse y atenerse a los marcos legales, éticos y deontológicos vigentes.

La prevención secundaria de la violencia de género es una tarea crucial en Atención Primaria. La detección y abordaje precoz por parte de los equipos resulta esencial para impedir consecuencias en la salud, y parte de una relación de confianza bien establecida, que garantiza la comodidad, la privacidad y la confidencialidad.

La detección de la violencia de género se basa principalmente en la entrevista, pudiendo apoyarse en diversos cuestionarios o escalas que la evalúan.

El abordaje temprano de la violencia de género supone una situación delicada que debe plantearse de forma individualizada, observando la actitud de la mujer hacia la misma en un proceso de cambio.

Los profesionales reconocen falta de formación sobre violencia de género, siendo una situación problemática que puede limitar o recortar su papel en la prevención secundaria, por lo que mejorar la formación resulta imprescindible para mejorar la situación.

La prevención de la violencia de género desde Atención Primaria debe contemplarse como un aspecto más en un marco de prevención global, en el que debe primar la coordinación interinstitucional (justicia, servicios sociales y sanitarios, administraciones locales, etc.)

\section{REFERENCIAS}

(1) Menéndez MI, Elipe P, Fernández E. Guía Clínica de Violencia de Género [Internet]. Madrid: Fisterra.com [actualizado 02 de Mayo de 2014; acceso 15 de Octubre de 2017]. Disponible en: http://www.fisterra.com/guias-clinicas/violencia-genero/ 
(2) WHO [Internet]. Ginebra: Organización Mundial de la salud; septiembre de 2016 [acceso el 03 de Octubre de 2017]. Centro de prensa: Violencia contra la mujer [aproximadamente 4 pantallas]. 4 Disponible http://www.who.int/mediacentre/factsheets/fs239/es/

(3) Cirici R, Querol N, Ripoll A. La consulta sanitaria: ¿un espacio privilegiado para la detección y el abordaje de la violencia de género? FMC [Internet] 2010: [acceso 27 Octubre de 2017]; 17(8): [550-559 Págs.] Disponible en: http://www.sciencedirect.com/science/article/pii/S1134207210702139

(4) Vinuesa MM, Farjas P, López RM, Peláez S, García S, López RM. Protocolo Común para la Actuación Sanitaria [Internet]. Madrid: Ministerio de Sanidad, Servicios Sociales e Igualdad; 2012 [acceso 03 de Octubre de 2017]. Disponible en: https://www.asturias.es/Astursalud/Ficheros/AS_Salud\%20Publica/AS_Promocion\%20 de $\% 20 l a \% 20$ Salud/Salud\%20de\%20las\%20mujeres\%20asturianas/Violencia\%20de\% 20g\%C3\%A9nero/PSanitarioVG2012.pdf

(5) Alonso F, Anaya F, Casals R, Gálvez F, Montesinos NM. Fundamentos teóricos de Enfermería comunitaria. 1. 1th ed. Madrid: Enfo ed. Para Fuden; 2007.

(6) Cadilhac DA, Sheppard L, Cumming TB, Thayabaranathan T, Pearce DC, Carter R, Maggus A. The health and economic benefits of reducing intimate partner violence: an Australian example. BMC Public Health [Internet] 2015 [acceso 8 de Octubre de 2017]; 15 (625): [7 Págs.] Disponible en: https://www.ncbi.nlm.nih.gov/pubmed/26155794

(7) Rodríguez GM, Vives C, Miralles JJ, San Sebastián M, Goicolea I. Detección de violencia de compañero íntimo en Atención Primaria de salud y sus factores asociados. Gac Sanit [Internet] 2016 [acceso 7 de Octubre de 2017]; [6Págs.]. Disponible en: http://www.elsevier.es/es-revista-gaceta-sanitaria-138-avance-resumendeteccion-violencia-companero-intimo-atencion-S0213911117300092?referer=buscador

${ }^{(8)}$ Molejón A, Muslera E, García z ML, González M, Martín D, Bruno J. Ámbito Sanitario del Protocolo Interdepartamental de Asturias de Asistencia a victimas de violencia de género del Principado [Internet]. Oviedo: Consejería del Instituto Asturiano de la Mujer; 2015 [acceso 03 de Octubre de 2017]. Disponible en: https://www.asturias.es/portal/site/astursalud/menuitem.2d7ff2df00b62567dbdfb51020 688a0c/?vgnextoid=396bf708c1674210VgnVCM10000097030a0aRCRD

(9) Gracia M. ¿Somos los profesionales sanitarios una barrera para la detección de la violencia de género? Enf Integ [Internet]. 2011 [acceso 22 de Octubre de 2017]; (93): [7 Páginas]. Disponible en: http://www.enfervalencia.org/ei/93/ENF-INTEG-93.pdf

(10) Cabrera M, Granero MJ. Enfermería, maltrato de género y presencia de menores: redescubriendo nuestro papel. Enferm. Glob [Internet] 2011 [acceso 27 de Octubre de 2017] ;(22): [10 Páginas]. Disponible en: http://revistas.um.es/eglobal/article/view/124021

(11) Macías AM. Conocimientos y Barreras de los profesionales sanitarios ante violencia de género. Doc. Enferm [Internet] 2016 [acceso 27 de Octubre de 2017]; (64): [23-25 Págs.]. Disponible http://www.colegiooficialdeenfermeriadehuelva.es/images/zoom/PTGAZN/viewsize/DocEnf_0 064.pdf

(12) Salcedo DM, Orchiucci P, Dias V, Yoshikawa E. ¿Cómo los profesionales de la Atención Primaria enfrentan la violencia contra las mujeres embarazadas? Enfermagem [Internet] 2014 [acceso el 08 de Octubre de 2017]; 22(3): [448-453]. Disponible en: https://www.ncbi.nlm.nih.gov/pubmed/25029056

${ }^{(13)}$ Cezar SM, Marques MJ, Filomena M. Representações sociais da violência contra a mulher na perspectiva da enfermagem. Interface [Internet] 2011 [acceso 12 de 
Octubre de 2017] 15 (37): [ 16 Pags]. Disponible en: http://www.scielo.br/scielo.php?script=sci_arttext\&pid=S1414-32832011000200007 (14) Valdés CA, García C, Sierra A. Violencia de género: conocimientos y actitudes de las enfermeras en atención primaria. Aten Primaria [Internet]. 2016 [acceso 23 de Octubre de 2017]; [10 páginas]. Disponible en: http://www.elsevier.es/pt-revista-atencionprimaria-27-articulo-violencia-genero-conocimientos-actitudes-las-S0212656716300385

(15) Juncal Plazaola J, Ruiz I, Hernández E. Validación de la versión corta del Woman Abuse Screening Tool para su uso en Atención Primaria en España. Gac Sanit [Internet] 2008 [acceso 04 de Octubre de 2017] 22(5): [415-420 Págs.] Disponible en: http://scielo.isciii.es/scielo.php?script=sci_arttext\&pid=S0213-

\section{5}

(16) Unidad de Apoyo e Investigación de la Escuela andaluza de salud pública. Catálogo de instrumentos para el cribado y frecuencia del maltrato físico, psicológico y sexual [Internet]. Observatorio de salud de la mujer. Granada: 2015 [acceso el 19 de Octubre de 2017]. Disponible en: https://www.msssi.gob.es/organizacion/sns/planCalidadSNS/pdf/equidad/genero_vg_01.pdf

(17) Ivethe C, Rivera S, Reidl LM, Garcia M. Violence in teenage Mexican couples through electronic/social media. Invest Psico. [Internet] 2017 [acceso 15 de Octubre de 2017]; 7(1): [2593-2605 Págs.]. Disponible en: http://www.sciencedirect.com/science/article/pii/S2007471917300054

(18) Organización colegial de Enfermería de España. Resolución 32- 89 del Código Deontológico de la Enfermería Española: artículo 53 y 55. Ginebra (Suiza): Revisión 2012.

(19) Linares J. Análisis e intervención de Enfermería en la Violencia de Género. Importancia del diagnóstico dentro del Equipo de salud. [Internet] En: III Congreso para el estudio de la violencia contra las mujeres. Granada: Junta de Andalucía, Consejería de Justicia e Interior; 2012. 5 Págs. Disponible en: http://www.violenciageneroasistenciavictimas.es/index.php/lineas-de-actuacion/congresovg/iii-congreso/articulos-cientifico-tecnicos/136-analisis-e-intervencion-de-enfermeria-en-laviolencia-de-genero-importancia-del-diagnostico-dentro-del-equipo-de-salud

${ }^{(20)}$ Holgado M, Molina S, Pérez R. Plan de mejora para el abordaje de la violencia contra la mujer en el área de urgencias. Biblioteca Lascasas, 2015; 11(3). Disponible en: http://www.index-f.com/lascasas/documentos/lc0843.php 